\title{
Accuracy analysis of a single-fault Markov model for FADEC system
}

\author{
CAI Jing*, HU Wei, and CAI Kunye \\ College of Civil Aviation, Nanjing University of Aeronautics and Astronautics, Nanjing 211106, China
}

\begin{abstract}
Time-limited dispatching (TLD) analysis of the full authority digital engine control (FADEC) systems is an important part of the aircraft system safety analysis and a necessary task for the certification of commercial aircraft and aeroengines. In the time limited dispatch guidance document ARP5107B, a single-fault Markov model (MM) approach is proposed for TLD analysis. However, ARP5107B also requires that the loss of thrust control (LOTC) rate error calculated by applying the single-fault MM must be less than $5 \%$ when performing airworthiness certification. Firstly, the sources of accuracy errors in three kinds of MM are analyzed and specified through a case study of the general FADEC system, and secondly a two-fault MM considering maintenance policy is established through analyzing and calculating the expected repair time when two related faults happen. Finally, a specific FADEC system is given to study on the influence factors of accuracy error in the single-fault MM, and the results show that the accuracy error of the single-fault MM decreases with the increase of short or long prescribed dispatch time, and the range values of short time (ST) and long time (LT) are determined to satisfy the requirement of accuracy error within $5 \%$.
\end{abstract}

Keywords: full authority digital engine control (FADEC) system, Markov model (MM), accuracy analysis, loss of thrust control (LOTC).

DOI: $10.21629 / J S E E .2019 .05 .20$

\section{Introduction}

Full authority digital engine control (FADEC) systems are very important to control the engine thrust from the beginning of fuel metering to the point of fuel shutoff. Therefore FADEC systems are designed around a dual channel control system, and incorporate a degree of redundancy. Timelimited dispatch (TLD) came into existence following the introduction of FADEC systems to the Boeing 767 aircraft, and TLD allows the dispatch of aircraft with faults present

Manuscript received October 15, 2018.

*Corresponding author.

This work was supported by the National Natural Science Foundation of China (51705242), Shanghai Sailing Program (16YF1404900) and the Fundamental Research Funds for the Central Universities (NS2015072). for a predetermined length of time when a redundant unit of the system fails, which means that some unscheduled failures are unnecessary to be corrected before the next flight; therefore the corresponding flight delay is avoided and aircraft dispatch ability increases [1-5]. However, the maximum time allowed for keeping the fault must be addressed; therefore, TLD analysis has been a necessary work of compliance verification to apply for type certification of aircraft or engine with FADEC systems, according to these airworthiness terms, such as Part 25.1309 "Equipment, Systems and Installation", Part 33.28 "Engine Control Systems" and Part 33.75 "System Safety" of the Federal Aviation Regulations [6,7].

In the policy letter ANE-1993-328 TLD-R1, only the time weighted average (TWA) method and the Markov model (MM) method are currently adopted to analyze TLD [8-10]. The Monte Carlo method has many advantages [11-16], but it is not mature enough to be adopted by the industry. The analysis accuracy of the TWA method is lower than that of the MM but the TWA method is widely used because of its easy operation [17]. Especially as the number of components increases in the FADEC system, the number of states of the system will increase exponentially, which makes the modeling process complicated or impossible, so the MM method is hard to be applied. In order to make the MM method for widespread adoption, the single fault condition is only considered in the MM method to approximate the loss of thrust control (LOTC) rate of the engine, i.e., a single-fault MM shows that only single fault conditions as individual states, and all secondary faults from the single states lead to the LOTC state. In reality, the situation that multiple faults exist simultaneously in the FADEC system is always not to be considered, because in the case of one fault occurrence, the probability of the occurrence of another fault during the following dispatch is relatively low due to the characteristics of the high repair rate and the low failure rate of the aero engine. Therefore the single-fault MM is always acceptable and applicable in 
the industry. However, according to the policy letter ANE1993-328TLD-R1 in Appendix B of SAE ARP5107B, the accuracy error of the single-fault MM should be proved to be within 5\% if the single-fault MM method is applied for TLD analysis [18]. Therefore, the accuracy of TLD analysis is a widely concerned problem. Therefore, the main purpose of this research is to provide a method to analyze the sources of accuracy error in single-fault MM and verify the magnitude of errors.

There have been some research on the accuracy of TLD analysis. Prescott and Andrews at Loughborough University proposed the Monte Carlo simulation (MCS) method instead of the single-fault MM method, and with some example, concluded that the MCS method was much more accurate than the single-fault MM method [10]. Based on the MCS method, Lu et al. [11] put forward a TLD analysis method of dispatch with multiple faults to determine the maintenance interval. It is undeniable that the MCS method has great advantages in solving multi-failure problems in TLD analysis, but the MCS is not the recommended method in SAE ARP5107B and should be required to demonstrate its compliance. Furthermore, the MCS method is only applied in the simplified FADEC system with three or four components, which is far from the actual complex FADEC system [11-13]. Therefore, whether the MCS method can be used in TLD analysis of the actual complex FADEC system or not should be further verified.

According to SAE ARP5107B, it has shown that the single-fault MM is the most suitable method for TLD analysis of the actual complex FADEC system so far, but it is a challenge to prove that the accuracy error of the singlefault MM is within 5\%. Therefore, the sources of accuracy error and its influence factors in the MM method will be analyzed in this study. The remainder of this paper is organized as follows. The single-fault MM method is investigated in Section 2. The sources of accuracy error in the MM method is analyzed and specified in Section 3. Then, a specific FADEC system is studied on the influence factors of accuracy error in the single-fault MM in Section 4, and the conclusions and suggestions for future research are presented in Section 5.

\section{Single-fault MM}

\subsection{TLD faults}

There are four dispatch categories that relate to TLD faults [19,20]:

(i) No dispatch (ND) state

A fault resulting in that the instantaneous LOTC rate exceeds 100 failures per $10^{6}$ flight hours (FHs) is categorized as the ND state. Since the hydromechanical/mechanical
(HM) components in the FEDAC system have no redundancy, their faults are also categorized as ND faults, and their failure rate is indicated by $\lambda_{\mathrm{HM}}$.

(ii) Short time dispatch (STD) state

A fault resulting in that the instantaneous LOTC rate is between 75 and 100 failures per $10^{6}$ FHs is categorized as the STD state, and the STD state must be restored within the prescribed time, namely short time (ST), indicated by $\mathrm{ST}$, so the corresponding repair rate is $\frac{1}{\mathrm{ST}}$.

(iii) Long time dispatch (LTD) state

A fault not resulting in that the instantaneous LOTC rate exceeds 75 failures per $10^{6} \mathrm{FHs}$ is categorized as the LTD state, and the LTD state must be restored within the prescribed time, namely long time (LT), indicated by LT so the corresponding repair rate is $\frac{1}{\mathrm{LT}}$.

(iv) Uncovered (UC) state

A fault that is ignored in the reliability analysis is categorized as the UC state, which also causes the LOTC event. According to the statistics, the UC state accounts for $0 \%$ to $5 \%$ of the total failure. Therefore in practice, the UC failure rate can be expressed by

$$
\lambda_{\mathrm{UC}}=X \times \sum_{i} \lambda_{i}
$$

where $X$ is a coefficient $(X=0-0.05)$ and $\sum_{i} \lambda_{i}$ is the total failure rate of the FEDAC system.

\subsection{Instantaneous LOTC rate}

The LOTC rate of the engine is defined as

$$
\lambda_{\text {LOTC }}=\frac{\text { Probability flow into the } P(\text { LOTC }) \text { state }}{1-P(\text { LOTC })} .
$$

The single-fault MM shows only single-fault conditions as individual states, and all second faults from the single states lead to the LOTC state. Therefore, a generic singlefault MM for the FADEC system is illustrated in Fig. 1, including the following three states: full-up, fault and LOTC.

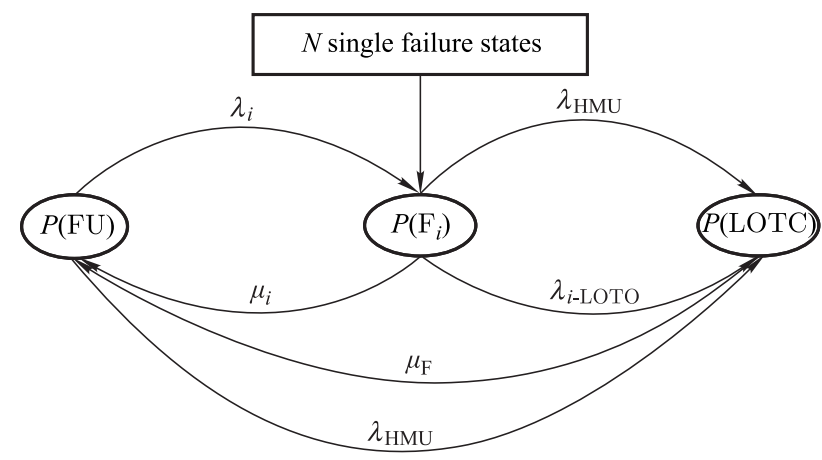

Fig. 1 Single-fault MM 
According to the theory of the MM, the following equation can be obtained:

$$
P(\mathrm{FU})+P(\mathrm{LOTC})+\sum_{i}^{N} P\left(\mathrm{~F}_{i}\right)=1
$$

where $P(\mathrm{FU}), P\left(\mathrm{~F}_{i}\right)$, and $P(\mathrm{LOTC})$ represent the probabilities of being in the full-up, fault, and LOTC states, respectively.

From the Markov state transition, the following equations can be drawn:

$$
\begin{gathered}
\frac{\mathrm{d} P\left(\mathrm{~F}_{i}\right)}{\mathrm{d} t}=\lambda_{i} P(\mathrm{FU})-\left(\mu_{i}+\lambda_{i-\mathrm{LOTC}}+\lambda_{\mathrm{HMU}}\right) P\left(\mathrm{~F}_{i}\right) \\
\frac{\mathrm{d} P(\mathrm{LOTC})}{\mathrm{d} t}=\lambda_{\mathrm{HMU}} P(\mathrm{FU})+ \\
\left(\lambda_{i-\text { LOTC }}+\lambda_{\mathrm{HMU}}\right) P\left(\mathrm{~F}_{i}\right)-\mu_{\mathrm{F}} P(\text { LOTC })
\end{gathered}
$$

When the system reaches a steady state, the probability of the system flowing into and out of each state is equal to 0 , so

$$
\frac{\mathrm{d} P\left(\mathrm{~F}_{i}\right)}{\mathrm{d} t}=\frac{\mathrm{d} P(\text { LOTC })}{\mathrm{d} t}=0 .
$$

Based on (4) - (6), the average LOTC rate of the singlefault MM is given by

$$
\begin{gathered}
\lambda_{\mathrm{LOTC}}=\frac{\lambda_{\mathrm{HMU}}}{1.0+\sum\left\{\lambda_{i} /\left(\mu_{i}+\lambda_{i-\mathrm{LOTC}}+\lambda_{\mathrm{HMU}}\right)\right\}}+ \\
\frac{\sum\left\{\lambda_{i} \times\left(\lambda_{i-\mathrm{LOTC}}+\lambda_{\mathrm{HMU}}\right) /\left(\mu_{i}+\lambda_{i-\text { LOTC }}+\lambda_{\mathrm{HMU}}\right)\right\}}{1.0+\sum\left\{\lambda_{i} /\left(\mu_{i}+\lambda_{i-\mathrm{LOTC}}+\lambda_{\mathrm{HMU}}\right)\right\}}
\end{gathered}
$$

where $\lambda_{i}$ is the failure rate of the $i$ th component. $\mu_{\mathrm{F}}$ is the repair rate for the LOTC state. $\mu_{i}(i=1, \ldots, N)$ is the repair rate for ST and LT failures, equal to the reciprocal of the ST and LT repair intervals. $\lambda_{\mathrm{HMU}}$ is the failure rate of the mechanical/hydraulic failure and uncovered failure (HMU), and $\lambda_{\mathrm{HMU}}=\lambda_{\mathrm{HM}}+\lambda_{\mathrm{UC}} \cdot \lambda_{i-\mathrm{LOTC}}$ is the average failure rate of the system due to a second fault when operating in a fault dispatch configuration.

\section{Sources of accuracy error in MM}

The accuracy error of the single-fault MM occurs due to ignoring concurrence of multiple failures conditions in the single-fault MM. Therefore, in order to quantitatively illustrate the accuracy error of the LOTC rate, a general FADEC system is assumed as shown in Fig. 2. Because only the sources of accuracy error in the MM are researched in this study, in order to simplify the analysis, it is assumed that all units in the general FADEC system are the same, with a failure rate $\lambda$.

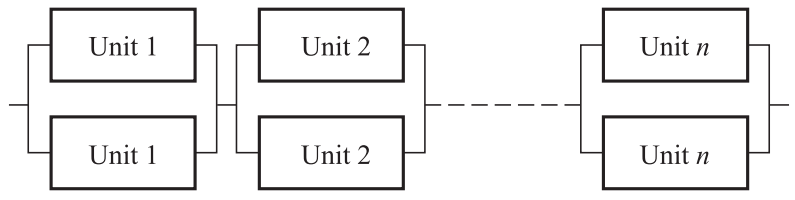

Fig. 2 Configuration of general FADEC system

\subsection{Single-fault MM}

The transfer diagram of the single-fault MM can be illustrated as Fig. 3 shows.

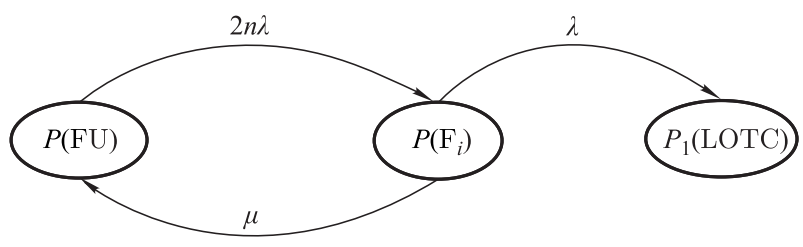

Fig. 3 Transfer diagram of single-fault MM

Accordingly to Fig. 3, the probability of the system in the LOTC state can be expressed as

$$
P_{1}(\mathrm{LOTC})=n \lambda P\left(\mathrm{~F}_{i}\right) .
$$

\subsection{Two-fault MM}

A two-fault MM shows not only single fault conditions but also two faults conditions as individual states. Therefore, the transfer diagram of the two-fault MM can be illustrated in Fig. 4.

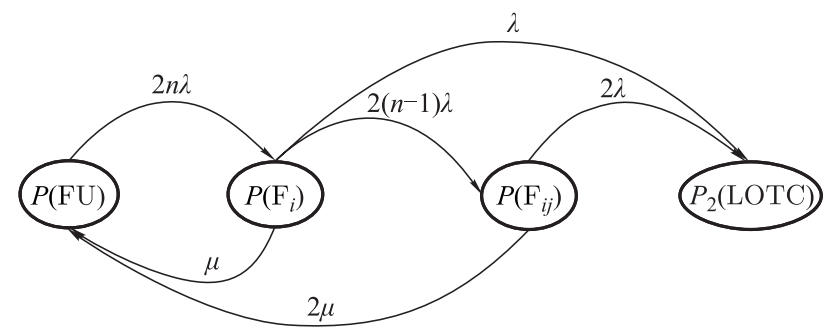

Fig. 4 Transfer diagram of two-fault MM

According to Fig. 4, the probability of the system in the LOTC state can be expressed as

$$
\begin{gathered}
P_{2}(\mathrm{LOTC})=n \lambda P\left(\mathrm{~F}_{i}\right)+2 n \lambda P\left(\mathrm{~F}_{i j}\right) \\
P\left(\mathrm{~F}_{i j}\right)=\frac{2(n-1) \lambda P\left(\mathrm{~F}_{i}\right)}{2 \mu+2 \lambda} .
\end{gathered}
$$

Substitute (10) into (9) and we can obtain

$$
P_{2}(\mathrm{LOTC})=\left(\frac{2(n-1) \lambda^{2}}{\mu+\lambda}+\lambda\right) n P\left(\mathrm{~F}_{i}\right)
$$




\subsection{Three-fault MM}

A three-fault MM should consider single-fault conditions, two-fault conditions and three-fault conditions at the same time. Therefore, the transfer diagram of the three-fault MM can be illustrated in Fig. 5 .

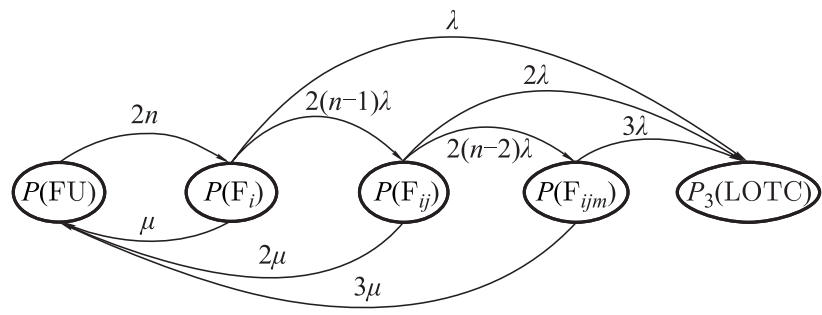

Fig. 5 Transfer diagram of three-fault MM

According to Fig. 5, the probability of the system in the LOTC state can be expressed as

$$
\begin{gathered}
P_{3}(\mathrm{LOTC})=n \lambda P\left(\mathrm{~F}_{i}\right)+2 n \lambda P\left(\mathrm{~F}_{i j}\right)+3 n \lambda P\left(\mathrm{~F}_{i j m}\right) \\
P\left(\mathrm{~F}_{i j}\right)=\frac{(n-1) \lambda P\left(\mathrm{~F}_{i}\right)}{\mu+\lambda+(n-2) \lambda} \\
P\left(\mathrm{~F}_{i j m}\right)=\frac{2 n(n-2) \lambda P\left(\mathrm{~F}_{i j}\right)}{3 \mu+3 \lambda}
\end{gathered}
$$

Substitute (13) and (14) into (12) and we can obtain

$$
\begin{gathered}
P_{3}(\text { LOTC })=\left(\lambda+\frac{2(n-1) \lambda^{2}}{\mu+\lambda+(n-2) \lambda}+\right. \\
\left.\frac{2(n-2) \lambda}{\mu+\lambda} \times \frac{(n-1) \lambda^{2}}{\mu+\lambda+(n-2) \lambda}\right) n P\left(\mathrm{~F}_{i}\right) .
\end{gathered}
$$

\subsection{Accuracy analysis}

The error without considering the two-fault condition in the single-fault MM is

$$
d_{12}=\frac{P_{2}(\mathrm{LOTC})-P_{1}(\mathrm{LOTC})}{P_{1}(\mathrm{LOTC})}=\frac{2(n-1) \lambda}{\mu+\lambda} .
$$

The error without considering three-fault conditions in the two-fault MM is

$$
\begin{gathered}
d_{23}=\frac{P_{3}(\mathrm{LOTC})-P_{2}(\mathrm{LOTC})}{P_{2}(\mathrm{LOTC})}= \\
\frac{\frac{2(n-2) \lambda}{\mu+\lambda} \times \frac{(n-1) \lambda^{2}}{\mu+\lambda+(n-2) \lambda}}{\frac{2(n-1) \lambda^{2}}{\mu+\lambda}+\lambda}+ \\
\frac{\frac{2(n-1) \lambda^{2}}{\mu+\lambda+(n-2) \lambda}-\frac{2(n-1) \lambda^{2}}{\mu+\lambda}}{\frac{2(n-1) \lambda^{2}}{\mu+\lambda}+\lambda} .
\end{gathered}
$$

In the actual FADEC system, the failure rate of the unit is generally about $10^{-6} \mathrm{FH}^{-1}$. Therefore, the variation of $d_{12}$ and $d_{23}$ with the number of components $n$ and the repair rate $\mu$, can be obtained respectively in Fig. 6 and Fig. 7.

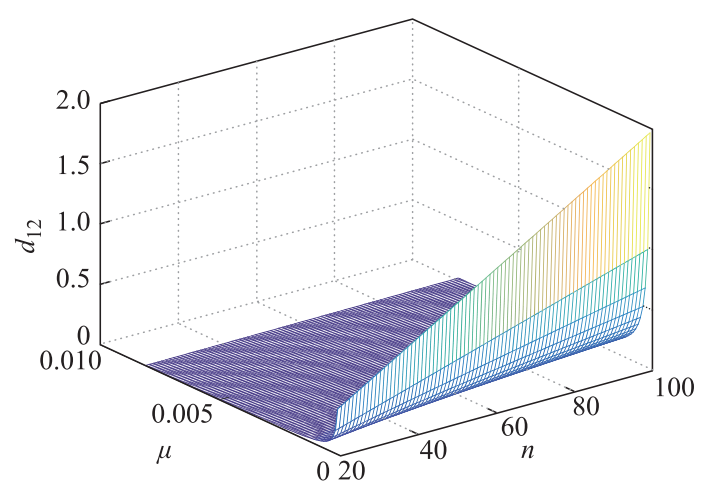

(a) The $d_{12}$ variation with $n$ and $\mu$

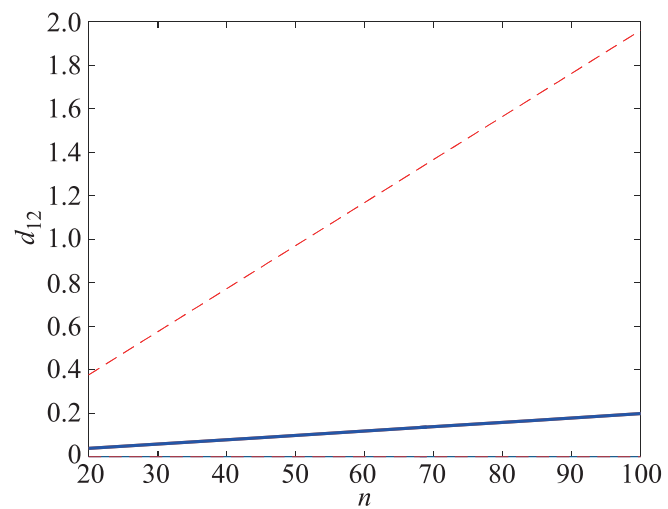

(b) The $d_{12}$ variation with $n$ in given $\mu$ - : $\mu=1.0 \mathrm{e}^{-03} ; \quad---: \mu=1.0 \mathrm{e}^{-04}$.

Fig. 6 The $d_{12}$ variation

It is shown in Fig. 6 that $d_{12}$ increases with the decrease of the repair rate and the increase of the number of components in the FADEC system. For example, $d_{12}$ reaches $4.2 \%$ when $n=25$ and $\mu=1.0 \mathrm{e}-03$, as shown in Fig. 6(b). In practice, $\mu$ may be smaller than $1.0 \mathrm{e}-03$ and $n$ is larger than 25 , so the actual accuracy error is always greater than $4.2 \%$. For example, $d_{12}$ reaches $9.6 \%$ when $n=50$ and $\mu=1.0 \mathrm{e}-03$. Therefore, whether the single-fault MM can meet the accuracy requirement in Appendix B of SAE ARP5107B depends on $\mu$ and $n$ in the actual FADEC system.

It is shown in Fig. 7 that $d_{23}$ fluctuates increasingly with the decrease of the repair rate and the increase of $n$. However, $d_{23}$ is very small, close to 0 . Therefore, three and more faults conditions do not need to be considered in the MM to calculate the LOTC rate, and the two-fault MM can be regarded as an approximate method to get an exact result for TLD analysis. 


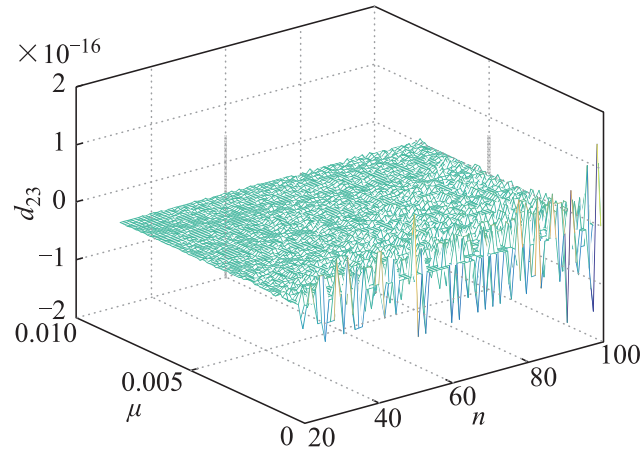

(a) The $d_{23}$ variation with $n$ and $\mu$

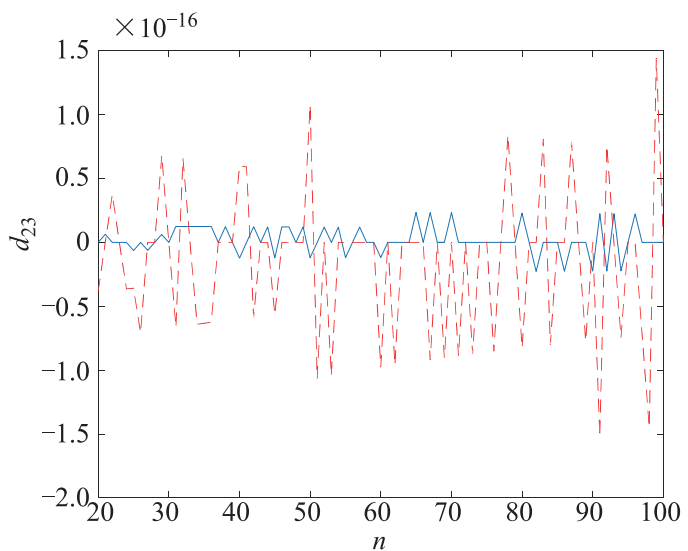

(b) The $d_{23}$ variation with $n$ in given $\mu$ - : $\mu=1.0 \mathrm{e}-03 ; \quad---: \mu=1.0 \mathrm{e}-04$.

Fig. 7 The $d_{23}$ variation

\section{Influence of ST and LT on accuracy error in single-fault $\mathrm{MM}$}

In the two-fault MM, when two related faults happen and one of them reaches the expiration of dispatch firstly, there are two choices: (i) the two faults are repaired together, (ii) only the expired fault is repaired [21-23]. The first choice would always be carried out in airlines because it can save the repair cost due to the existing economic dependence. Therefore, the first choice will be considered in this study to analyze the influence of the repair rate on the accuracy error in the single-fault MM with a specific FADEC system.

There are 85 faults in the specific FADEC system and the partial fault tree is shown in Fig. 8. VBV means variable bleed valve, TBV means transient bleed valve, and VSV means variable stator vane.

According to the principle of dispatch categories in SAE ARP5107B and the fault tree, the 85 faults can be divided into $10 \mathrm{ND}$ faults, $2 \mathrm{ST}$ faults and $73 \mathrm{LT}$ faults. Therefore, there are 75 dispatch faults, and the LOTC rate caused by single fault and two faults, respectively represented by $\lambda_{i_{-} L}(i=1,2, \ldots, 75)$ and $\lambda_{i j_{-} L}(i, j=1,2, \ldots, 75, i \neq$ $j$ ), can be obtained based on the fault tree.

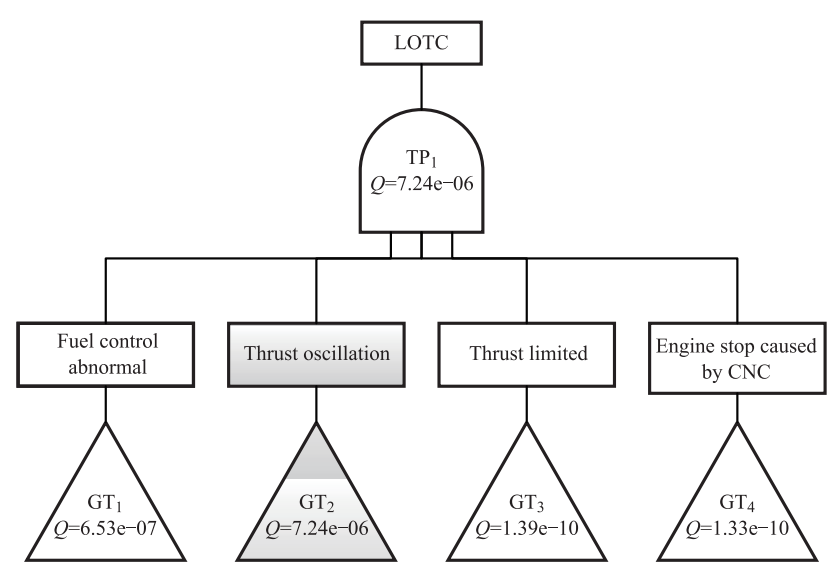

(a) Main fault tree

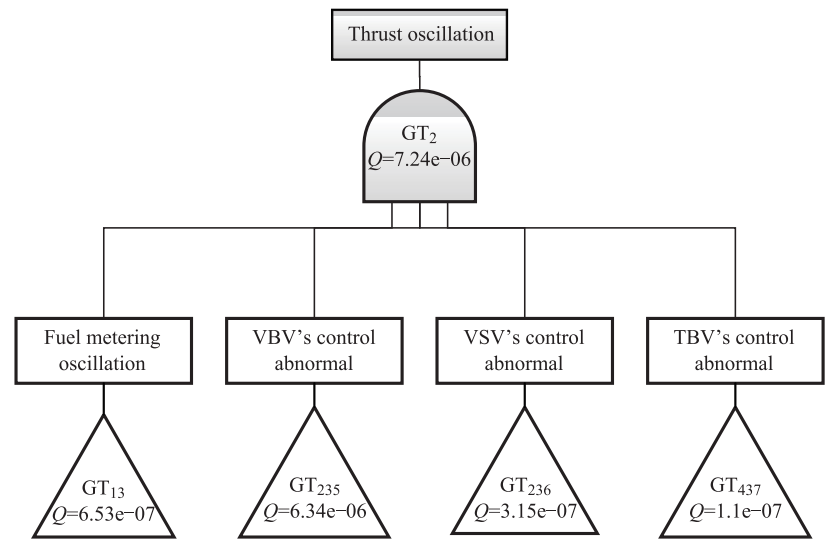

(b) $\mathrm{GT}_{2}$ subtree

Fig. 8 Partial fault tree diagram

According to the two-fault MM, it is known that there are four states: full-up, single fault, two faults and LOTC, and $P(\mathrm{FU}), P\left(\mathrm{~F}_{i}\right), P\left(\mathrm{~F}_{i j}\right)$ and $P($ LOTC) represent the probabilities of being in the full-up, single fault, two faults and LOTC states respectively. The two-fault MM can be illustrated in Fig. 9.

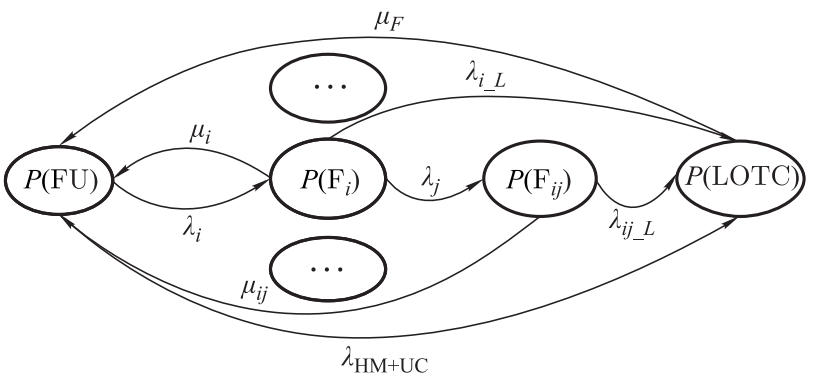

Fig. 9 Two-fault MM

According to Fig. 9, in order to calculate the LOTC rate, firstly the equations about $P(\mathrm{FU}), P\left(\mathrm{~F}_{i}\right), P\left(\mathrm{~F}_{i j}\right)$ and $P$ (LOTC) should be set up respectively. 


\subsection{Equations about $P\left(\mathrm{~F}_{i}\right)$}

Accordingly to Fig. $9, P\left(\mathrm{~F}_{i}\right)(i=1,2, \ldots, 75)$ can be expressed as

$$
\begin{gathered}
\frac{\mathrm{d} P\left(\mathrm{~F}_{i}\right)}{\mathrm{d} t}=P(\mathrm{FU}) \lambda_{i}-\left(\mu_{i}+\sum_{j} \lambda_{j}+\lambda_{i_{-} L}\right) P\left(\mathrm{~F}_{i}\right), \\
i, j=1,2, \ldots, 75 ; i \neq j .
\end{gathered}
$$

There are two ST faults and 73 LT faults in the specific FADEC system. Therefore, there are 75 differential equations about $P\left(\mathrm{~F}_{i}\right)$.

\subsection{Equations about $P\left(\mathrm{~F}_{i j}\right)$}

According to Fig. 9, $P\left(\mathrm{~F}_{i j}\right)$ can be expressed as

$$
\begin{gathered}
\frac{\mathrm{d} P\left(\mathrm{~F}_{i j}\right)}{\mathrm{d} t}=P\left(\mathrm{~F}_{i}\right) \lambda_{j}-\left(\mu_{i j}+\lambda_{i j_{-} L}\right) P\left(\mathrm{~F}_{i j}\right), \\
i, j=1,2, \ldots, 75 ; i \neq j .
\end{gathered}
$$

Because ST faults and LT faults have different repair rates, the different sequences of two faults happening would result in different maximum fault duration time. Therefore, when both fault $i$ and fault $j$ happen, the expected repair time $\mu_{i j}$ should be determined as the following three cases:

Case 1 The ST fault $i$ happens first.

It is assumed that another ST/LT fault $j$ happens $\tau$ later after the fault $i$. Because $\frac{1}{\mu_{S}}$ is less than $\frac{1}{\mu_{L}}$, the two faults must be repaired in time $\frac{1}{\mu_{i j}}=\frac{1}{\mu_{S}}-\tau$, as shown in Fig. 10.

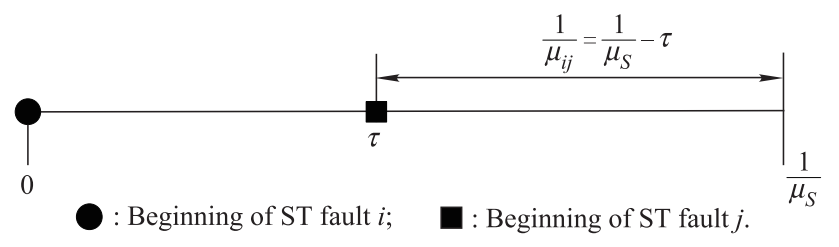

Fig. 10 ST fault happens first

Accordingly to Fig. 10, the expected repair time $\mathrm{E}\left(\frac{1}{\mu_{i j}}\right)$ can be obtained as follows:

$$
\begin{aligned}
& \mathrm{E}\left(\frac{1}{\mu_{i j}}\right)=\int_{0}^{1 / \mu_{S}} \lambda_{j} \mathrm{e}^{-\lambda_{j} \tau}\left(\frac{1}{\mu_{S}}-\tau\right) \mathrm{d} \tau= \\
& \frac{1}{\mu_{S}} \cdot \int_{0}^{1 / \mu_{S}} \lambda_{j} \mathrm{e}^{-\lambda_{j} \tau} \mathrm{d} \tau-\int_{0}^{1 / \mu_{S}} \lambda_{j} \tau \mathrm{e}^{-\lambda_{j} \tau} \mathrm{d} \tau= \\
& \frac{1}{\mu_{S}} \cdot \int_{0}^{1 / \mu_{S}} \lambda_{j} \mathrm{e}^{-\lambda_{j} \tau} \mathrm{d} \tau-\int_{0}^{1 / \mu_{S}} \lambda_{j} \tau \mathrm{e}^{-\lambda_{j} \tau} \mathrm{d} \tau=
\end{aligned}
$$

$$
\begin{gathered}
\frac{1}{\mu_{S}} \cdot\left(-\left.\mathrm{e}^{-\lambda_{j} \tau}\right|_{0} ^{1 / \mu_{S}}\right)+\int_{0}^{1 / \mu_{S}} \tau \mathrm{de}^{-\lambda_{j} \tau}= \\
\frac{1}{\mu_{S}} \cdot\left(1-\mathrm{e}^{-\frac{\lambda_{j}}{\mu_{S}}}\right)+\left.\tau \mathrm{e}^{-\lambda_{j} \tau}\right|_{0} ^{1 / \mu_{S}}-\int_{0}^{1 / \mu_{S}} \mathrm{e}^{-\lambda_{j} \tau} \mathrm{d} t= \\
\frac{1}{\mu_{S}} \cdot\left(1-\mathrm{e}^{-\frac{\lambda_{j}}{\mu_{S}}}\right)+\frac{1}{\mu_{S}} \cdot \mathrm{e}^{-\frac{\lambda_{j}}{\mu_{S}}}+\left.\frac{1}{\lambda_{j}} \mathrm{e}^{-\lambda_{j} \tau}\right|_{0} ^{1 / \mu_{S}}= \\
\frac{1}{\mu_{S}}-\frac{1}{\lambda_{j}}+\frac{1}{\lambda_{j}} \mathrm{e}^{-\frac{\lambda_{j}}{\mu_{S}}}
\end{gathered}
$$

Case 2 Both of the faults are LT faults.

Similar to Case 1 , the expected repair time $\mathrm{E}\left(\frac{1}{\mu_{i j}}\right)$ can be expressed by

$$
\mathrm{E}\left(\frac{1}{\mu_{i j}}\right)=\frac{1}{\mu_{L}}-\frac{1}{\lambda_{j}}+\frac{1}{\lambda_{j}} \mathrm{e}^{-\frac{\lambda_{j}}{\mu_{L}}} .
$$

Case 3 LT fault $i$ happens first and the ST fault $j$ happens after $\tau$.

Case 3 can be described in Fig. 11. If the ST fault happens during $\left(0, \frac{1}{\mu_{L}}-\frac{1}{\mu_{S}}\right)$, obviously the two faults should be repaired in time $\frac{1}{\mu_{S}}$, as shown in Fig. 11(a). If the ST fault happens during $\left[\frac{1}{\mu_{L}}-\frac{1}{\mu_{S}}, \frac{1}{\mu_{L}}\right)$, the two faults must be repaired in time $\frac{1}{\mu_{L}}-\tau$, as shown in Fig. 11(b).

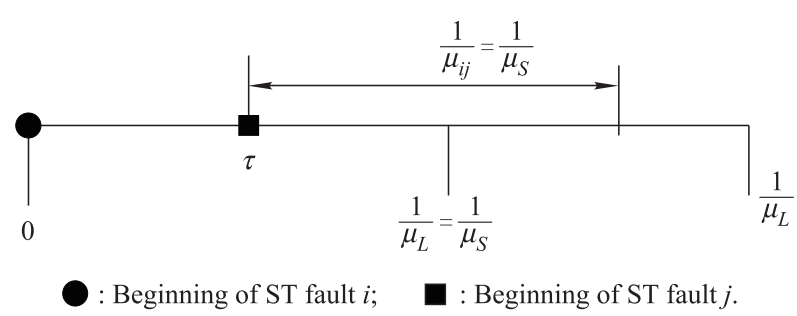

(a) ST fault happens during $\left(0, \frac{1}{\mu_{L}}-\frac{1}{\mu_{S}}\right)$

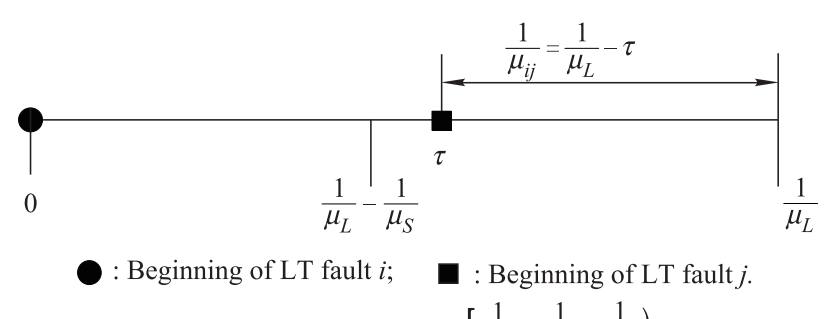

(b) ST fault happens during $\left[\frac{1}{\mu_{L}}-\frac{1}{\mu_{S}}, \frac{1}{\mu_{L}}\right)$

Fig. 11 LT fault $i$ happens first and ST fault $\boldsymbol{j}$ happens after $\tau$

Therefore, the expected repair time $\mathrm{E}\left(\frac{1}{\mu_{i j}}\right)$ can be obtained as follows:

$$
\mathrm{E}\left(\frac{1}{\mu_{i j}}\right)=\int_{0}^{1 / \mu_{L}-1 / \mu_{S}} \lambda_{j} \mathrm{e}^{-\lambda_{j} \tau} \cdot \frac{1}{\mu_{S}} \mathrm{~d} \tau+
$$




$$
\begin{aligned}
& \int_{1 / \mu_{L}-1 / \mu_{S}}^{1 / \mu_{L}} \lambda_{j} \mathrm{e}^{-\lambda_{j} \tau} \cdot\left(\frac{1}{\mu_{L}}-\tau\right) \mathrm{d} \tau= \\
& \frac{1}{\mu_{S}} \cdot \int_{0}^{1 / \mu_{L}-1 / \mu_{S}} \lambda_{j} \mathrm{e}^{-\lambda_{j} \tau} \mathrm{d} \tau+ \\
& \frac{1}{\mu_{L}} \cdot \int_{1 / \mu_{L}-1 / \mu_{S}}^{1 / \mu_{L}} \lambda_{j} \mathrm{e}^{-\lambda_{j} \tau} \mathrm{d} \tau-\int_{1 / \mu_{L}-1 / \mu_{S}}^{1 / \mu_{L}} \lambda_{j} \mathrm{e}^{-\lambda_{j} \tau} \cdot \tau \mathrm{d} \tau= \\
& \frac{1}{\mu_{S}} \cdot\left(-\left.\mathrm{e}^{-\lambda_{j} \tau}\right|_{0} ^{1 / \mu_{L}-1 / \mu_{S}}\right)+\frac{1}{\mu_{L}} \cdot\left(-\left.\mathrm{e}^{-\lambda_{j} \tau}\right|_{1 / \mu_{L}-1 / \mu_{S}} ^{1 / \mu_{L}}\right)+ \\
& \int_{1 / \mu_{L}-1 / \mu_{S}}^{1 / \mu_{L}} \tau \mathrm{de}^{-\lambda_{j} \tau}= \\
& \frac{1}{\mu_{S}}-\frac{1}{\mu_{S}} \cdot \mathrm{e}^{\frac{\lambda_{j}}{\mu_{S}}-\frac{\lambda_{j}}{\mu_{L}}}+\frac{1}{\mu_{L}} \cdot \mathrm{e}^{\frac{\lambda_{j}}{\mu_{S}}-\frac{\lambda_{j}}{\mu_{L}}}- \\
& \frac{1}{\mu_{L}} \cdot \mathrm{e}^{-\frac{\lambda_{j}}{\mu_{L}}}+\left.\tau \mathrm{e}^{-\lambda_{j} \tau}\right|_{1 / \mu_{L}-1 / \mu_{S}} ^{1 / \mu_{L}}-\int_{1 / \mu_{L}-1 / \mu_{S}}^{1 / \mu_{L}} \mathrm{e}^{-\lambda_{j} \tau} \mathrm{d} t= \\
& \frac{1}{\mu_{S}}-\frac{1}{\mu_{S}} \cdot \mathrm{e}^{\frac{\lambda_{j}}{\mu_{S}}-\frac{\lambda_{j}}{\mu_{L}}}+\frac{1}{\mu_{L}} \cdot \mathrm{e}^{\frac{\lambda_{j}}{\mu_{S}}-\frac{\lambda_{j}}{\mu_{L}}}-\frac{1}{\mu_{L}} \cdot \mathrm{e}^{-\frac{\lambda_{j}}{\mu_{L}}}+ \\
& \frac{1}{\mu_{L}} \cdot \mathrm{e}^{-\frac{\lambda_{j}}{\mu_{L}}}-\left(\frac{1}{\mu_{L}}-\frac{1}{\mu_{S}}\right) \cdot \mathrm{e}^{\frac{\lambda_{j}}{\mu_{S}}-\frac{\lambda_{j}}{\mu_{L}}}+\left.\frac{1}{\lambda_{j}} \mathrm{e}^{-\lambda_{j} \tau}\right|_{1 / \mu_{L}-1 / \mu_{S}} ^{1 / \mu_{L}}= \\
& \frac{1}{\mu_{S}}+\frac{1}{\lambda_{j}} \mathrm{e}^{-\frac{\lambda_{j}}{\mu_{L}}}-\frac{1}{\lambda_{j}} \mathrm{e}^{\frac{\lambda_{j}}{\mu_{S}}-\frac{\lambda_{j}}{\mu_{L}}} .
\end{aligned}
$$

There are two ST faults and 73 LT faults. Therefore, there are 5550 kinds of two-fault combinations. However, some combinations result in the high LOTC rate, greater than $1.0 \mathrm{e}-05 \mathrm{FH}^{-1}$, and even constitute the cut set resulting in LOTC directly. Therefore, only 5020 kinds of two-fault combinations are selected eventually. Therefore, there are 5020 differential equations about $P\left(\mathrm{~F}_{i j}\right)$.

\subsection{Equations about $P(\mathrm{FU})$}

Accordingly to Fig. 9, $P(\mathrm{FU})$ can be expressed as

$$
\begin{gathered}
\frac{\mathrm{d} P(\mathrm{FU})}{\mathrm{d} t}=\sum_{i} \mu_{i} \cdot P\left(\mathrm{~F}_{i}\right)+\sum_{i} \sum_{i \neq j}\left(\mu_{i j} \cdot P\left(\mathrm{~F}_{i j}\right)\right)+ \\
P(\mathrm{LOTC}) \cdot \mu_{F}-\left(\lambda_{\mathrm{HMU}}+\sum_{i} \lambda_{i}\right) \cdot P(\mathrm{FU}), \\
i, j=1,2, \ldots, 75 ; i \neq j .
\end{gathered}
$$

\subsection{Equations about $P($ LOTC)}

\begin{tabular}{|c|c|c|c|c|}
\hline \multirow{2}{*}{ Equation } & \multicolumn{4}{|c|}{ Coefficient } \\
\hline & $P\left(\mathrm{~F}_{i}\right)$ & $P\left(\mathrm{~F}_{i j}\right)$ & $P($ LOTC $)$ & $P(\mathrm{FU})$ \\
\hline $\begin{array}{c}\text { No. } 1-75 \\
(i, j=1,2, \ldots, 75 ; i \neq j)\end{array}$ & $-\left(\mu_{i}+\sum_{j} \lambda_{j}+\lambda_{i_{-} L}\right)$ & 0 & 0 & $\lambda_{i}$ \\
\hline $\begin{array}{c}\text { No. } 76-5095 \\
(i, j=1,2, \ldots, 75 ; i \neq j)\end{array}$ & 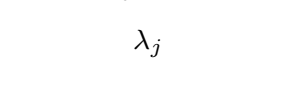 & $-\left(\mu_{i j}+\lambda_{i j_{-} L}\right)$ & 0 & 0 \\
\hline No. 5096 & $\sum_{i} \mu_{i}$ & $\stackrel{\mu_{i j}}{(i, j=1,2, \ldots, 75 ; i \neq j)}$ & $\mu_{F}$ & $-\left(\lambda_{\mathrm{HMU}}+\sum_{i} \lambda_{i}\right)$ \\
\hline No. 5097 & $\sum_{i} \lambda_{i_{-} L}$ & $\begin{array}{c}\lambda_{i j_{-} L} \\
(i, j=1,2, \ldots, 75 ; i \neq j)\end{array}$ & $-\mu_{F}$ & $\lambda_{\mathrm{HMU}}$ \\
\hline
\end{tabular}

According to Fig. 9, P(LOTC) can be expressed as

$$
\begin{gathered}
\frac{\mathrm{d} P(\mathrm{LOTC})}{\mathrm{d} t}=\sum_{i} \lambda_{i_{-} L} \cdot P\left(\mathrm{~F}_{i}\right)+\lambda_{\mathrm{HMU}} \cdot P(\mathrm{FU})+ \\
\sum_{i} \sum_{i \neq j}\left(\lambda_{i j_{-} L} \cdot P\left(\mathrm{~F}_{i j}\right)\right)-\mu_{F} \cdot P(\mathrm{LOTC}) \\
i, j=1,2, \ldots, 75 ; i \neq j .
\end{gathered}
$$

According to the above analysis, there are 5097 equations in total. When the system reaches a steady state, all flows into one state and out of a state have the same probability, i.e., all the differential equations are equal to 0 , so

$$
\frac{\mathrm{d} P\left(\mathrm{~F}_{i}\right)}{\mathrm{d} t}=\frac{\mathrm{d} P\left(\mathrm{~F}_{i j}\right)}{\mathrm{d} t}=\frac{\mathrm{d} P(\mathrm{FU})}{\mathrm{d} t}=\frac{\mathrm{d} P(\text { LOTC })}{\mathrm{d} t}=0 .
$$

Therefore, the simultaneous equations can be established and their coefficients are shown in Table 1.

Table 1 Coefficients of simultaneous equations

Through the simultaneous equations, $P($ LOTC) can be obtained and the LOTC rate can be expressed as

$$
\lambda_{\mathrm{LOTC}}=\frac{\mu_{F} P(\text { LOTC })}{1-P(\text { LOTC })} .
$$

$\mu_{F}$ is the rate at which the system returns from the LOTC state to its intact state, and $\mu_{F}$ has no effect on the LOTC rate, so it is always assumed that $\mu_{F}$ is equal to 1 . (i) Set ST $=125 \mathrm{FH}$, i.e., $\mu_{S}=0.008 \mathrm{FH}^{-1}$

The change of the LOTC rate with LT can be obtained in Fig. 12. It can be known in Fig. 12 that because twofault conditions considered in the two-fault MM contribute to the increased LOTC rate, the LOTC rate of the two-fault MM is greater than that of the single-fault MM. For example, LT should be less than $1126 \mathrm{FH}$ in the two-fault MM to control the LOTC rate within $1.0 \mathrm{e}-05 \mathrm{FH}^{-1}$, and 
LT should be less than $1285 \mathrm{FH}$ in the single-fault MM, which accords with the actual situation.

Fig. 12 tells that the difference of the LOTC rate between the two-fault MM and the single-fault MM is getting bigger and bigger with LT increasing, i.e., the accuracy error of single-fault MM increases with LT, as shown in Fig. 13. The accuracy error is up to $5 \%$ when LT exceeds $2005 \mathrm{FH}$. Therefore in this case, LT should be less than $2005 \mathrm{FH}$ if the single-fault MM is applied in TLD analysis.

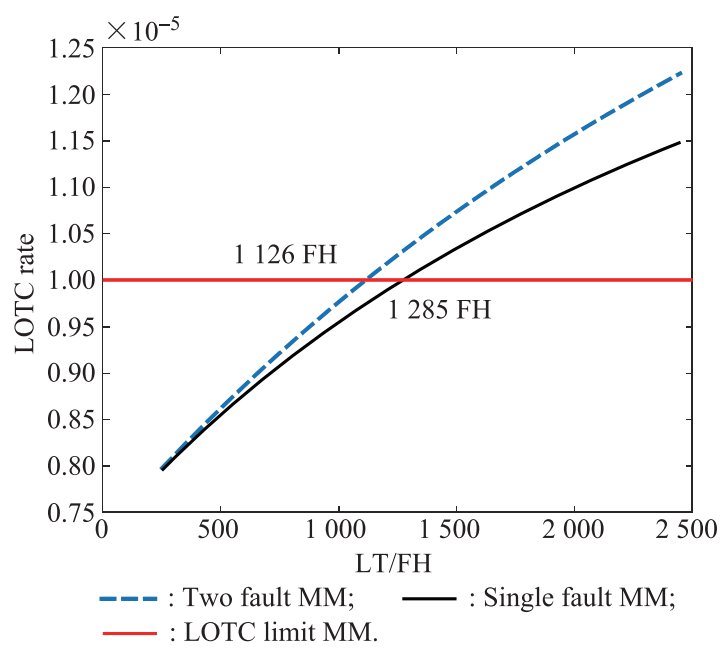

Fig. 12 Change of LOTC rate with LT when ST=125 FH

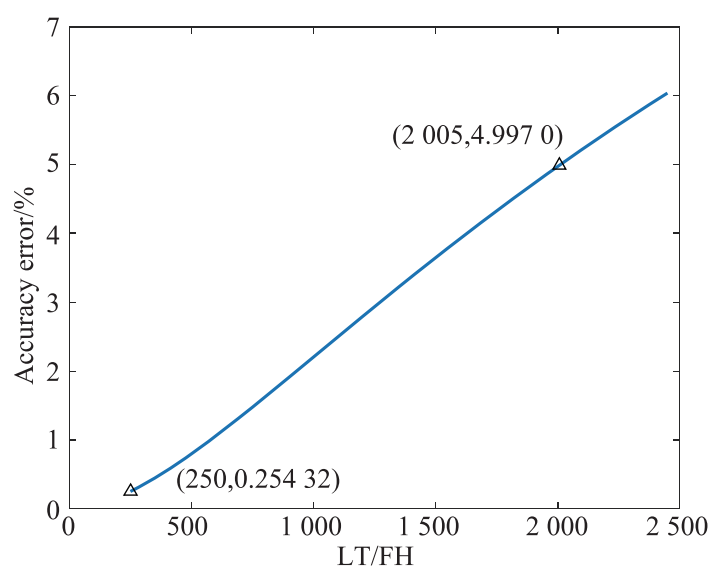

Fig. 13 Accuracy error when ST=125 FH

(ii) Set ST $=250$ FH, i.e., $\mu_{S}=0.004 \mathrm{FH}^{-1}$

The change of the LOTC rate with LT can be obtained in Fig. 14, which is similar to Fig. 12. LT should be less than $1273 \mathrm{FH}$ in the single-fault MM to control the LOTC rate within $1.0 \mathrm{e}-05 \mathrm{FH}^{-1}$.

As shown in Fig. 15, the accuracy error of single-fault MM is up to 5\% when LT exceeds $1999 \mathrm{FH}$. Therefore, when ST=250 FH, LT must be fewer than $1999 \mathrm{FH}$ to ensure that the single-fault MM can be applied in TLD analysis.

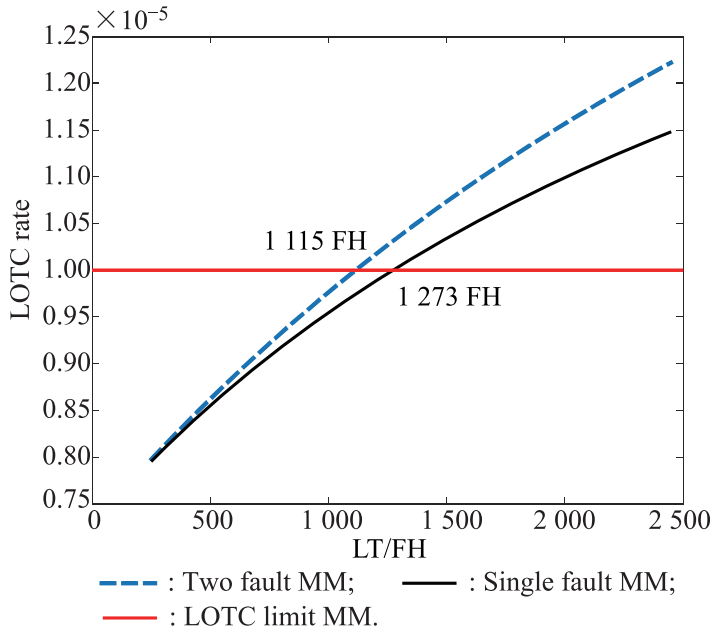

Fig. 14 Change of LOTC rate with LT when ST=250 FH

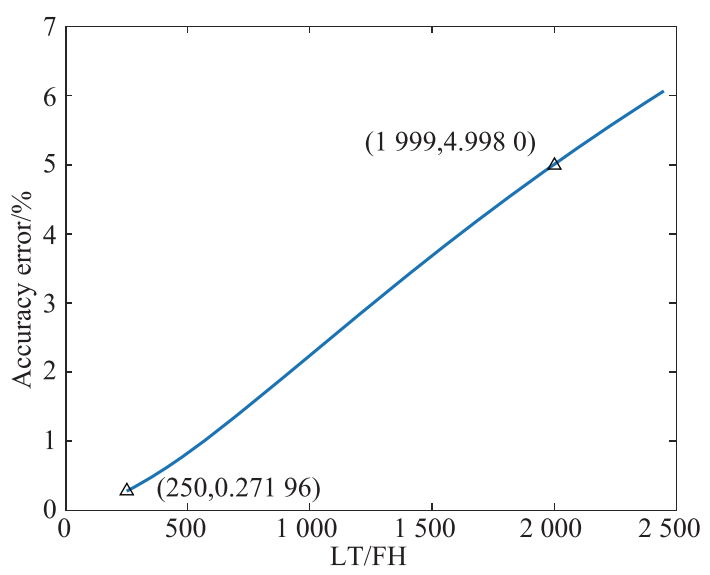

Fig. 15 Accuracy error when ST=250 FH

Through the analysis above, it is known that the accuracy error of the single-fault MM increases with ST and LT. When ST is determined, the value of LT that results in the LOTC rate exceeding $1.0 \mathrm{e}-05 \mathrm{FH}^{-1}$ is greater than the value of LT required to control the accuracy error within $5 \%$. For example, $1285<2005$ when $\mathrm{ST}=125 \mathrm{FH}$, and $1273<1999$ when ST=250 FH. Therefore, for the specific FADEC system, the single-fault MM can satisfy the requirement of accuracy error in the policy letter ANE1993-328TLD-R1. Furthermore, within the range values of ST and LT, the functional relationship between ST, LT and LOTC rate based on the single-fault MM can be determined to further obtain optimal ST and LT to enhance the engine dispatch rate and decrease the downtime.

\section{Conclusions}

A case study of the general FADEC system is given to analyze the sources of accuracy errors in the MM, and the results show that three and more faults conditions do not need to be considered in the MM to calculate the LOTC 
rate, and the two-fault MM can be regarded as an approximate method to get an exact result for TLD analysis. Then a specific FADEC system is given to study the accuracy error of the single-fault MM, and the results show that:

(i) The accuracy error of the single-fault MM decreases with the increase of ST and LT.

(ii) The determined range values of ST and LT can satisfy the requirement of the accuracy error within 5\%.

In further study, the influence of the number of ST or LT faults on the accuracy error of the single-fault MM will be investigated, and the effect of different maintenance policies on TLD analysis will be studied.

\section{References}

[1] HJELMGREN K, SVENSSON S, HANNIUS O. Reliability analysis of a single-engine aircraft FADEC. Proc. of the International Symposium on Product Reliability and Maintainability, 1998: $401-407$.

[2] CHEN B, LI C, LI Y, et al. Reliability analysis method of an aircraft engine FADEC system. Proc. of the 8th International Conference on Reliability, Maintainability and Safety, 2009: $289-292$.

[3] PRESCOTT D R, ANDREWS J D. Monte Carlo simulation modelling of aircraft dispatch with known faults. Proc. of the 8th International Conference on Reliability, Maintainability and Safety, 2009: $532-535$.

[4] PRESCOTT D R. Safety modelling for the time limited dispatch of aircraft. Leicestershire, UK: Loughborough University, 2007.

[5] BENEDA K. Development of a modular FADEC for small scale turbojet engine. Proc. of the 14th International Symposium on Applied Machine Intelligence and Informatics, 2016: $51-56$.

[6] SAE International Group. ARP5107B guidelines for timelimited-dispatch (TLD) analysis for electronic engine control systems. Washington, D.C.: Society of Automotive Engineers, 2006. https://saemobilus.sae.org/content/ARP5107B/.

[7] SAE International Group. ARP4761 guidelines and methods for conducting the safety assessment process on civil airborne system and equipment. Washington, D.C.: Society of Automotive Engineers, 1996. https://saemobilus.sae.org/ content/ARP4761/.

[8] CROWE D, CROM E. Improving the reliability of TF50, TF40, ETF40B FADEC. Proc. of the Turbine Technical Conference and Exposition. 2012: 439-443.

[9] BINEID M, FIELDING J P. Development of a civil aircraft dispatch reliability prediction methdology. Aircraft Engineering and Aerospace Technology, 2003, 75(6): 588-594.

[10] PRESCOTT D R, ANDREWS J D. Aircraft safety modeling for time-limited dispatch. Proc. of the Annual Reliability and Maintainability Symposium, 2005: 139-145.

[11] LU Z, RONG X, ZHOU J. TLD analysis method of dispatch with multiple faults based on Monte Carlo simulation for FADEC system. Acta Aeronautica et Astronautica Sinica. 2015, 36(12): 3970 - 3979.

[12] LU Z, LIANG X, ZUO M J, et al. Markov process based time limited dispatch analysis with constraints of both dispatch reliability and average safety levels. Reliability Engineering \& System Safety, 2017, 167: 84-94.

[13] LU Z, ZHOU J, LI X. Monte Carlo simulation based time limited dispatch analysis with the constraint of dispatch reliability for electronic engine control systems. Aerospace Science and Technology, 2018, 72: $397-408$.
[14] YAN F. A method on time-limited dispatch for the control system of civil aero-engine. Science Technology and Engineering, 2014, 14(28): 148 - 158. (in Chinese)

[15] CAO C, SHEN J, LUO M C, et al. FWorks: an integrated digital simulation platform for FADEC systems. Proc. of the Chinese Control Conference, 2017: 10316-10321.

[16] DING S T, QIU T, LIU X F, et al. FHA method for VBV position control function of FADEC system based on aero-engine dynamic model. Proc. of the 2nd International Symposium on Aircraft Airworthiness, 2011: 567-579.

[17] PRESCOTT D R, ANDREWS J D. A comparison of modelling approaches for the time-limited dispatch (TLD) of aircraft. Journal of Risk and Reliability, 2006, 220(1): 9-20.

[18] MEMORANDUM FAA. ANE-1993-33.28TLD-R1, policy for time-limited dispatch (TLD) of engines fitted with full authority digital engine control systems. Warrendale, Pennsylvania: Federal Aviation Administration, 1993. http://fsims.faa.gov/PICDetail.aspx?docId=PL-045.

[19] PRESCOTT D R, ANDREWS J D. Modeling and specification of time-limited dispatch categories for commercial aircraft. Journal of Dynamic Systems Measurement and Control, 2008, 130: 21004-21009.

[20] PRESCOTT D R, ANDREWS J D. The safe dispatch of aircraft with known faults. International Journal of Perform Ability Engineering, 2008, 4(3): 243-253.

[21] BAI J, ZHAO P, WANG W. TLD maintenance strategies of FADEC system. Maintenance, 2012, 6: 35 - 37. (in Chinese)

[22] BOUSSEMART M, LIMNIOS N, BICKARD T. Maintenance optimization of a digital engine control system with limit failure rate constrain. Proc. of the 22nd Congress of International Council of the Aeronautical Sciences, 2000: 621.1-621.10.

[23] ZHANG T H, LU Z, ZHOU J, et al. Operation interval decision method for dual redundant systems operating with faults. Aeronautical Computing Technique, 2015, 45(5): 33-36. (in Chinese)

\section{Biographies}

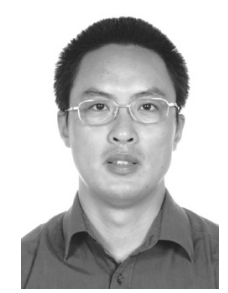

CAI Jing was born in 1976. He received his B.S. and $\mathrm{Ph}$.D. degrees from Nanjing University of Aeronautics and Astronautics in 1999 and 2007. He is currently an associate professor in Nanjing University of Aeronautics and Astronautics (NUAA). His research interests include reliability statistics, maintenance theory, and prognostic and health management (PHM)

E-mail: caijing@nuaa.edu.cn

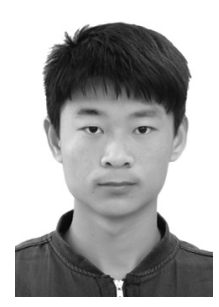

HU Wei was born in 1995. He is studying for his master's degree at Nanjing university of Aeronautics and Astronautics. His research interests include airworthiness, reliability and time-limited dispatch. E-mail: luanwu@nuaa.edu.cn

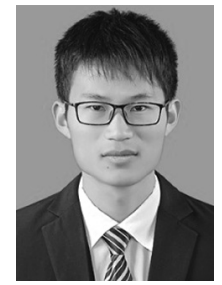

CAI Kunye was born in 1993. He is studying for his master's degree at Nanjing university of Aeronautics and Astronautics. His research interests include analytical application of quick access recorder data and continuous airworthiness management of civil aircraft.

E-mail: 852344855@163.com 